\title{
Algorithm for Term Linearizations of Aggregate Queries with Comparisons
}

\author{
Victor Felea ${ }^{1}$ and Violeta Felea ${ }^{2}$ \\ 1 CS Department, Al.I. Cuza University, Iasi, Romania \\ felea@infoiasi.ro \\ 2 FEMTO-ST, Franche-Comté University, Besançon, France \\ violeta.felea@femto-st.fr
}

\begin{abstract}
We consider the problem of rewriting queries based exclusively on views. Both queries and views can contain aggregate functions and include arithmetic comparisons. To study the equivalence of a query with its rewriting query, the so called "linearizations of a query" need to be computed. To find the linearizations of a query, the linearizations of terms from the query need to be generated. We propose an algorithm to find these term linearizations and give a bound for its time-complexity.
\end{abstract}

\section{Introduction}

The problem of rewriting queries using views $(R Q V)$ is expressed, informally, as follows: given $Q$ a query over a database schema, and $\mathcal{V}$ a set of view definitions, is it possible to answer the query $Q$ using only the answers to the views from $\mathcal{V}$ ? One of the application fields of RQV concerns extracting and gathering information on the environment, based on sensor networks. Small inexpensive devices, namely sensors, communicate information to collecting points, called sinks or base stations. In this context, of information collection out of several sources (stored in views), located on sensor nodes, expressing queries using views. In [7, the authors apply the notions of query interface for data integration to sensor networks.

In case of finding a query $Q^{\prime}$, corresponding to a given query $Q$, and expressed by the views from $\mathcal{V}$, such that $Q$ is equivalent to $Q^{\prime}$, the problem of $(R Q V)$ for the query $Q$ has a solution. This query $Q^{\prime}$ is called a rewriting query of $Q$.

The equivalence problem of two aggregate queries with comparisons was investigated in 4, 1 , 2, 5]. The equivalence problem of the two queries is achieved using the equivalence of their reduced queries. A reduced query associated to a query $Q$ uses the so-called "linearizations" (or complete ordering) of the set of all terms from $Q$.

In 3, query equivalence between view-based query and schema query, using linearizations, is proven; no linearization computation has been given. This is the main aim of this work: to propose an algorithm to compute linearizations. By our best knowledge, an algorithm to obtain all linearizations of terms from a query has not been proposed in the literature. 
In the paper, we consider the problem of generating all linearizations corresponding to a query that uses arithmetic comparisons and aggregate functions; we consider a motivated example (see section 2) and give an algorithm that computes these linearizations, and its time complexity in section 3 . We finally conclude.

\section{Motivating Example}

Let us give an example about a schema regarding sensor networks used in statistical applications concerning the weather. The network nodes are organized in clusters [6], one common architecture used in sensor networks.

Example 1. We assume there exist sensors for the following type of data: humidity, mist and temperature. One sensor can retrieve unique or multiple environmental data, e.g. temperature and humidity, or only temperature values. Sensors are placed in several locations, and have an identifier, a type, and they are grouped by regions. The type of a sensor characterizes the set of data types that it senses. A location is identified by two coordinates lat, long, where lat is the latitude and long is the longitude. A region has an identifier and a unique name. Let us consider the relational schema $\mathcal{S}$ consisting of $\{R E G, P O I N T$, $S E N S O R, D A T A\}$, where $R E G$ represents regions and has $i d R e g$ as region identifier, and regName as region name; POINT represents the locations from the regions, and uses the $i d R e g$ attribute as the region identifier, and the lat, long attributes as the coordinates of a location from the region identified by $i d R e g$; SENSOR represents information about sensors with $i d S$ the sensor identifier, lat, long - the sensor coordinates, type $S$ its type related to the sensing capacity (e.g. type $S$ equals 1 for temperature and humidity, 2 for temperature and mist, 3 for humidity and mist, and so on), and $\operatorname{chS}$ designs if the sensor is a cluster head (its value equals 1 or 2 ). We assume here that the type of the sensors that can sense temperature equals either 1 or 2 ; DATA represents the values registered by sensors, and has the following attributes: $i d S$ - the sensor identifier, year, month, day, period - the date of the sensed information, the daily frequency of sensed data, expressed in seconds, and temp, humid, mist - the temperature, humidity, and mist values, respectively.

Let us consider the following views:

$V_{1}$ : Compute all pairs of the form (idReg, regName), where idReg is the region identifier, and regName is the name of the region identified by idReg, such that in the region $i d R e g$ at least a cluster head exists.

$V_{2}$ : Compute the maximum temperature for each region, each network location, and for every year.

Let us consider the following queries:

$Q_{1}$ : Find the maximum of temperature values for the "NORTH" region, between 2003 and 2010, considering data sensed only by cluster head sensors.

$Q_{2}$ : Find the maximum temperature corresponding to the "NORTH" region, on September 22nd, 2009. 
Example 2. Let us consider the schema, queries, and views from Example 1. We denote the attributes $i d R e g$, regName from $R E G$ by $x, y_{1}$, respectively, $i d R e g$, lat, long from POINT by $y_{2}, y_{3}, y_{4}$, and $i d S$, lat, long, typeS, chS from SENSOR by $z_{1}, z_{2}, z_{3}, t_{1}, t_{2}$, and the attributes from the schema DATA by $z_{5}, z_{6}, z_{7}, z_{8}, z_{9}, t, z_{11}, z_{12}$. To be short, we denote by $D, R, S, P$ the relational symbols DATA, REG, SENSOR, POINT, respectively. Let $c_{1}=$ "NORTH", $c_{2}=2003$ and $c_{3}=2010$. To obtain a condition associated to $Q_{1}$, we replace the variables $y_{1}, t_{2}$ by $c_{1}, 1$, respectively. Since $y_{2}=x, z_{2}=y_{3}, z_{3}=y_{4}$, and $z_{5}=z_{1}$, the query $Q_{1}$ is expressed as the following condition:

$Q_{1}: h(x, \max (t)) \leftarrow D\left(z_{1}, z_{6}, z_{7}, z_{8}, z_{9}, t, z_{11}, z_{12}\right) \wedge R\left(x, c_{1}\right) \wedge S\left(z_{1}, y_{3}, y_{4}, t_{1}, 1\right) \wedge$ $P\left(x, y_{3}, y_{4}\right) \wedge C$, where $C \equiv\left(\left(t_{1}=1\right) \vee\left(t_{1}=2\right)\right) \wedge\left(z_{6} \geq c_{2}\right) \wedge\left(z_{6} \leq c_{3}\right)$.

The query $Q_{2}$, and the views $V_{1}, V_{2}$ defined in Example 1, have similar expressions.

\section{Term Linearizations of Aggregate Queries}

In this section we specify some notions about the term linearizations and give an algorithm to compute these linearizations constructed by the terms from the conditions contained in the aggregate query. For other notions, see [3].

Two aggregate queries $Q_{1}$ and $Q_{2}$ having identical heads are said equivalent, if their answers are equal, i.e. $Q_{1}(D)=Q_{2}(D)$, for any database $D$ defined on Dom. Let $f_{i}$ be the body of $Q_{i}$. Since each $f_{i}$ is a disjunction of relational atoms and of an expression constructed from comparison atoms using the conjunction and the disjunction operators, it can contain comparisons of terms. The comparisons of an expression $f_{i}$ induce, in general, a partial order among the constants and variables of the query. To study the equivalence of two queries, we need to consider linear orderings such that a given partial ordering of the set of terms is compatible with a set of linear orderings of the same set of terms.

The set of all terms from a query can be considered as a set of groups, where all attributes of a group correspond to the same value domain. Let $C$ be a set of comparison atoms, and $t_{1}<t_{2}$ be a comparison atom. We say that $C$ implies $t_{1}<t_{2}$, denoted $C \models t_{1}<t_{2}$, if for each substitution $\tau$, the statement $(\tau \delta=$ true for each $\delta \in C$ ) implies $\tau t_{1}<\tau t_{2}$. For a comparison atom having the form $t_{1}=t_{2}$ or $t_{1}>t_{2}$ the implication relation of a comparison atom from a set of comparison atoms is similar. Let $T$ be a set of terms (variables or constants) that are semantically equivalent. A linearization of $T$ is a set of comparisons $L$ having the terms in $T$ such that for any two different elements $t_{1}, t_{2}$ from $T$, exactly one of the following comparisons: $t_{1}<t_{2}, t_{2}<t_{1}$, or $t_{1}=t_{2}$ is implied by $L$. If $T$ has $t_{1}, \ldots, t_{p}$ as elements, let $\left(t_{1}^{\prime}, \ldots, t_{p}^{\prime}\right)$ be a permutation of the elements $t_{j}, 1 \leq j \leq p$. Then we can represent a linearization $L$ of $T$ as $t_{1}^{\prime} \rho_{1} t_{2}^{\prime} \rho_{2} \ldots t_{p-1}^{\prime} \rho_{p-1} t_{p}^{\prime}$, where the operators $\rho_{i}$ are ' $<$ ' or ' $=$ '. We say that a substitution $\tau$ from $\left\{t_{1}^{\prime}, \ldots, t_{p}^{\prime}\right\}$ into Dom, that preserves constants, satisfies the linearization $L$, denoted $\tau(L)=t r u e$, if for each $i, 1 \leq i<p, \tau t_{i}^{\prime}=\tau t_{i+1}^{\prime}$, when $\rho_{i}$ is ' $=$ ', and $\tau t_{i}^{\prime}<\tau t_{i+1}^{\prime}$, when $\rho_{i}$ is ' $<$ '. We denote by $t_{1}=t_{2}=\ldots=t_{h}$, the set of comparisons $t_{i}=t_{i+1}$ contained in $L$, for any $i, 1 \leq i<h$. Intuitively, 
a linearization is a list of different variables and constants such that between two elements of the list there exists one of the ' $=$ ' or ' $<$ ' operators. In this way, a linearization produces a partition of all terms into equivalence classes; an equivalence class contains all terms $t_{1}, \ldots, t_{h}$ such that $t_{1}=t_{2}=\ldots=t_{h}$.

We say that a set of comparisons $C$ is satisfiable, if there exists a substitution $\tau$ from terms into Dom such that $\tau$ satisfies all comparisons from $C$. We say that $\tau$ satisfies $C$ or $\tau(C)=t r u e$, if $\tau t_{1}<\tau t_{2}$ if $C \equiv t_{1}<t_{2}$, and $\tau t_{1}=\tau t_{2}$ if $C \equiv t_{1}=t_{2}$. The value of $\tau(C)$ is extended naturally to expressions $C$ obtained from basic comparisons using the conjunction and disjunction operators. We say that a linearization $L$ of $T$, and a set of comparisons $C$ are compatible, if $L \cup C$ is satisfiable.

In the following we give a method to obtain all linearizations of $T$, compatible with $C$. Let $C$ be the conjunction of the comparisons $t_{i} \sigma_{i} t_{i}^{\prime}$, i.e. $C=\left(t_{1} \sigma_{1} t_{1}^{\prime}\right) \wedge$ $\ldots \wedge\left(t_{h} \sigma_{h} t_{h}^{\prime}\right)$, where the operators $\sigma_{i}$ are relational operators. Let $C^{\prime}$ be the formula obtained from $C$, by replacing $\left(t_{i} \leq t_{j}\right)$ with $\left(t_{i}<t_{j}\right) \vee\left(t_{i}=t_{j}\right)$, and $\left(t_{i} \geq t_{j}\right)$ with $\left(t_{j}<t_{i}\right) \vee\left(t_{i}=t_{j}\right)$. Using for the expression $C^{\prime}$, the distributivity of the conjunction versus the disjunction, we obtain a formula $C^{\prime \prime}$ having the form: $C^{\prime \prime}=E_{1} \vee E_{2} \vee \ldots \vee E_{p}(1)$, where $E_{j}$ is a conjunction of comparisons of the form $t_{i}<t_{j}$ or $t_{i}=t_{j}$. In this disjunction, we take only consistent conjunctions by eliminating those conjunctions that are inconsistent, i.e. which contain at least two comparisons of the form: $t_{1}<t_{2}$ and $t_{1}=t_{2}$, or two comparisons of the form $t_{1}<t_{2}$ and $t_{2}<t_{1}$. Let $E$ be an arbitrary conjunction from $C^{\prime \prime}$. Associated to $E$, we construct a forest, denoted $G_{E}$. Firstly, for the set of all comparisons $S_{E}$ of the form $t_{i}=t_{j}$ from $E$, we take the transitive and symmetrical closure of $S_{E}$. This closure produces a set of equivalence classes $C l_{1}, \ldots, C l_{q}$. Let us denote by Term $1=\left\{t_{1}, \ldots, t_{p}\right\}$, the remainder of the variables and the constants from $E$. The forest $G_{E}$ associated to $E$ is defined as follows: its nodes are labeled with $C l_{j}, 1 \leq j \leq q$ and $t_{i}, 1 \leq i \leq p$. Let $\operatorname{Var}(C)$ be the set of variables from $C$ and Const $(C)$ the set of constants from $C$.

The edge set $\mathcal{N}$ of the forest $G_{E}$ is specified as follows:

- Let $t_{1}$ and $t_{2}$ be variables or constants. If $t_{1}<t_{2}$ appears in $E$, and $t_{1}$ and $t_{2}$ occur in Term 1 , then $\left(t_{1}, t_{2}\right) \in \mathcal{N}$.

- Let $C l_{j}$ be a class and $t_{2}$ from Term1. If there exists $t^{\prime} \in C l_{j}$ such that $t^{\prime}<t_{2}$ occurs in $E$, then $\left(C l_{j}, t_{2}\right) \in \mathcal{N}$.

- Let $t_{1}$ be from Term 1 , and $C l_{h}$ a class. If there exists $t^{\prime} \in C l_{h}$ such that $t_{1}<t^{\prime}$ occurs in $E$, then $\left(t_{1}, C l_{h}\right) \in \mathcal{N}$.

- Let $C l_{j}, C l_{h}$ be two different classes. If there exist the terms $t_{1}, t_{2}$, where $t_{1} \in C l_{j}$ and $t_{2} \in C l_{h}$ such that $t_{1}<t_{2}$ appears in $E$, then $\left(C l_{j}, C l_{h}\right) \in \mathcal{N}$.

Since the labels associated to different nodes contain disjoint sets of terms, we identify the nodes with their labels. If a class $C l_{j}$ consists of the elements $t_{1}, \ldots$, $t_{s}$, we denote it as $\left\{t_{1}, \ldots, t_{s}\right\}$ or $\left\{t_{1}=\ldots=t_{s}\right\}$.

Example 3. Let $C \equiv\left(x_{1} \leq x_{2}\right) \wedge\left(x_{2}>x_{3}\right) \wedge\left(y_{1} \leq x_{2}\right)$. The formula corresponding to $C$ has the form: $C^{\prime}=\left[\left(x_{1}<x_{2}\right) \vee\left(\left(x_{1}=x_{2}\right)\right] \wedge\left(x_{3}<x_{2}\right) \wedge\left[\left(y_{1}<\right.\right.\right.$ $\left.\left.x_{2}\right) \vee\left(y_{1}=x_{2}\right)\right]$. The expression $C^{\prime \prime}$ has the form: $C^{\prime \prime}=E_{1} \vee E_{2} \vee E_{3} \vee E_{4}$, where 
$E_{1}=\left(x_{1}<x_{2}\right) \wedge\left(x_{3}<x_{2}\right) \wedge\left(y_{1}<x_{2}\right), E_{2}=\left(x_{1}<x_{2}\right) \wedge\left(x_{3}<x_{2}\right) \wedge\left(y_{1}=x_{2}\right)$, $E_{3}=\left(x_{1}=x_{2}\right) \wedge\left(x_{3}<x_{2}\right) \wedge\left(y_{1}<x_{2}\right), E_{4}=\left(x_{1}=x_{2}\right) \wedge\left(x_{3}<x_{2}\right) \wedge\left(y_{1}=x_{2}\right)$.

The forests associated to each of the four expressions are given in the following figure, where nodes are labeled $\beta_{i}$.

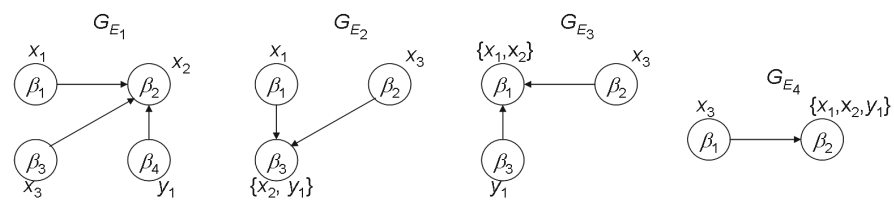

\subsection{A Method to Obtain the Linearizations of $C$}

In this subsection, we specify a method to obtain all linearizations of $\operatorname{Var}(C) \cup$ Const $(C)$ compatible with $C$ and corresponding to a forest $G_{E}$ associated to an expression $E$ from $C^{\prime \prime}$. The construction of this set of linearizations will be done recursively in function of the number of edges from $G_{E}$.

- The base step: Let $G_{E}$ having zero edges, and $\beta_{1}, \ldots, \beta_{p}$ its nodes. Let $i_{1}, \ldots, i_{p}$ be a permutation of the indexes $1,2, \ldots, p$. Let us denote by $\mathcal{M}_{G_{E}}^{L}$ the set of linearizations defined by $G_{E}$. For this base step, we take: $\mathcal{M}_{G_{E}}^{L}=\left\{\beta_{i_{1}} \sigma_{1} \beta_{i_{2}} \sigma_{2} \ldots \beta_{i_{p-1}} \sigma_{p-1} \beta_{i_{p}} \mid\left(i_{1}, \ldots, i_{p}\right)\right.$ is a permutation of $\{1,2$, $\left.\ldots, p\}, \sigma_{i} \in\left\{{ }^{\prime}<^{\prime},^{\prime}={ }^{\prime}\right\}\right\}$.

We make the following convention: $\left\{t_{1}=t_{2}=\ldots=t_{s}\right\}=\left\{t_{1}^{\prime}=t_{2}^{\prime}=\ldots=\right.$ $\left.t_{r}^{\prime}\right\}$ becomes: $\left\{t_{1}=t_{2}=\ldots=t_{s}=t_{1}^{\prime}=t_{2}^{\prime}=\ldots=t_{r}^{\prime}\right\}$.

- The inductive step: Assume that we have computed the set of linearizations corresponding to any forest $G$ having at most $n$ edges, where $n$ is a natural number. Let $G$ be a forest having $n+1$ edges. Let $t_{0}$ be an initial node from $G$, and $\gamma_{1}, \ldots, \gamma_{k}$ the immediate successors of $t_{0}$. Let $G^{\prime}$ be the forest obtained from $G$ by deleting the edges $\left(t_{0}, \gamma_{j}\right), 1 \leq j \leq k$, and deleting the node $t_{0}$. Since $G^{\prime}$ has at most $n$ edges, by induction we have computed $\mathcal{M}_{G^{\prime}}^{L}$. Using the linearizations of $G^{\prime}$, we compute the linearizations for $G$. Let $L \equiv s_{1}<s_{2}<\ldots<s_{m}$ be an element of $\mathcal{M}_{G^{\prime}}^{L}$, where $m$ is the number of the nodes of $G^{\prime}$. An element $s_{i}$ contains a set of terms from $\operatorname{Var}(C) \cup \operatorname{Const}(C)$. The label $\gamma_{j}$ belongs to a unique $s_{i}$, for each $j, 1 \leq j \leq k\left(\gamma_{j} \subseteq s_{i}\right)$. Let us denote by $i n d(j)$ this natural number $i$. Let $i_{0}$ be the minimum of $i n d(j)$, for each $j, 1 \leq j \leq k$. Now, we define a set of linearizations for $G$ corresponding to $L$ by inserting $t_{0}$ in $L$ before $s_{i_{0}}$. Let us denote by $\operatorname{Insert}\left(t_{0}, L, l\right)$ the linearizations obtained from $L$ by inserting $t_{0}$ between the positions $l$ and $l+1$. There are two linearizations in $\operatorname{Insert}\left(t_{0}, L, l\right)$ in case $l \geq 1$, denoted $L_{1}, L_{2}$, where $L_{1} \equiv s_{1}<s_{2}<\ldots<s_{l}<t_{0}<s_{l+1}<\ldots<s_{m}$, and $L_{2} \equiv s_{1}<s_{2}<\ldots<\left\{s_{l}=t_{0}\right\}<s_{l+1}<\ldots<s_{m}$. If $l=0$ the insertion takes place before $s_{1}$ and $\operatorname{Insert}\left(t_{0}, L, l\right)$ consists of one sequence, namely $L_{1}$, where $L_{1} \equiv t_{0}<s_{1}<s_{2}<\ldots<s_{m}$. For the linearization $L$ and the node $t_{0}$, we consider the union of the sets $\operatorname{Insert}\left(t_{0}, L, l\right)$, for each $l, 0 \leq l<i_{0}$. Let us denote this set by $\mathcal{M}\left(t_{0}, L\right)$. 
Now, we define the set of linearizations corresponding to $G$ as follows: $\mathcal{M}_{G}^{L}=$ $\left\{L^{\prime} \mid(\exists L)\left(L \in \mathcal{M}_{G^{\prime}}^{L}\right)\right.$ such that $\left.L^{\prime} \in \mathcal{M}\left(t_{0}, L\right)\right\}$.

Let $E_{j}$ be an expression from relation (1), $1 \leq j \leq p$. Let $G_{E_{j}}$ be the forest corresponding to $E_{j}$. Let $\mathcal{M}_{G_{E_{j}}}^{L}$ be the set of linearizations computed for the forest $G_{E_{j}}$. Let $\mathcal{M}(C)$ be the union of the $\mathcal{M}_{G_{E_{j}}}^{L}$ sets, for each $j, 1 \leq j \leq p$. Regarding these notations, we have the following results.

Lemma 1. Let $E$ be a consistent conjunction of comparisons of the form $t_{i}<$ $t_{j}$ or $t_{i}=t_{j}$. Let $L$ be a linearization compatible with $E$, and $G_{E}$ the forest associated to $E$. Then, we have $L \in \mathcal{M}_{G_{E}}^{L}$.

Proof. Using the induction on the number of the comparisons of the form ' $<$ ' from $E$ (this number is equal to the number of the edges from $G_{E}$ ).

Theorem 1. Let $L$ be a linearization of $\operatorname{Var}(C) \cup \operatorname{Const}(C)$ and $\mathcal{M}(C)$ the set of linearizations computed for $C$ as above. Then, we have: $L$ is compatible with $C$ iff $L \in \mathcal{M}(C)$.

Proof. Let $L$ be a linearization from $\mathcal{M}(C)$. There exists an integer $j, 1 \leq j \leq p$ such that $L \in \mathcal{M}_{G_{E_{j}}}^{L}$. The linearization $L$ can be represented as $C l\left(t_{1}\right)<C l\left(t_{2}\right)$ $<\ldots<C l\left(t_{m}\right)$, where $C l\left(t_{i}\right)$ are equivalence classes corresponding to $L$, and $t_{i}$ is an element from that class. A class $C l\left(t_{i}\right)$ consists of a single variable, a single constant, or a set of terms $\left\{t_{1}^{\prime}, \ldots, t_{h}^{\prime}\right\}, h \geq 2$, and this set contains at most one constant. The union of the classes $C l\left(t_{i}\right)$ is $\operatorname{Var}(C) \cup \operatorname{Const}(C)$. Let us define the substitution $\tau$ as follows: if $C l\left(t_{i}\right)=\left\{t_{1}^{\prime}, \ldots, t_{h}^{\prime}\right\}$, then $\tau t_{j}^{\prime}=\tau t_{l}^{\prime}$, $1 \leq j<l \leq h$. The substitution $\tau$ is extended to classes: $\tau C l\left(t_{j}\right)=\tau t_{j}$. For two classes, we take the values for $\tau$ such that if $C l\left(t_{i}\right)<C l\left(t_{j}\right)$, then $\tau t_{i}<\tau t_{j}$. In this manner, we have $\tau(L)$ is true. Using the method to construct $\mathcal{M}_{G_{E_{j}}}^{L}$, we obtain $\tau\left(E_{j}\right)$ is true, hence $\tau(C)$ is true. That means $L \cup C$ is satisfiable.

Conversely, let $L$ be a linearization compatible with $C$. It results that there exists an integer $j, 1 \leq j \leq p$ such that $L$ is compatible with $E_{j}$. Using Lemma 1, we obtain $L \in \mathcal{M}_{G_{E_{j}}}^{L}$, hence $L \in \mathcal{M}(C)$.

Using Theorem 1, we obtain an algorithm that computes the set of all linearizations for a conjunction of comparisons.

\subsection{Algorithm to Compute Linearizations of $\mathcal{M}(C)$}

Firstly, let us denote by Linearizations $\operatorname{Comp}(G, \mathcal{L})$ an algorithm that computes the set $\mathcal{L}$ of all linearizations corresponding to the forest $G$. Let us consider some notations used in this algorithm. We denote by $h$ the number of levels from $G$. The terminal nodes of $G$ are considered on level 1. Let $N_{j}$ be the set of all nodes on level $j, 1 \leq j \leq h$. We denote by $\mathcal{L}_{j}$ the set of all linearizations for nodes situated on the levels $l$ in $G$, where $l \leq j$. We use the notation $\mathcal{M}(\gamma, L)$ for a set of linearizations obtained by inserting the node $\gamma$ into a linearization $L$, as specified in subsection 3.1. By $\mathcal{L}_{j, l}$ we denote a set of linearizations of nodes 
from $G$, that is a subset of $\mathcal{L}_{j}$. The algorithm proceeds level by level, beginning with the first level.

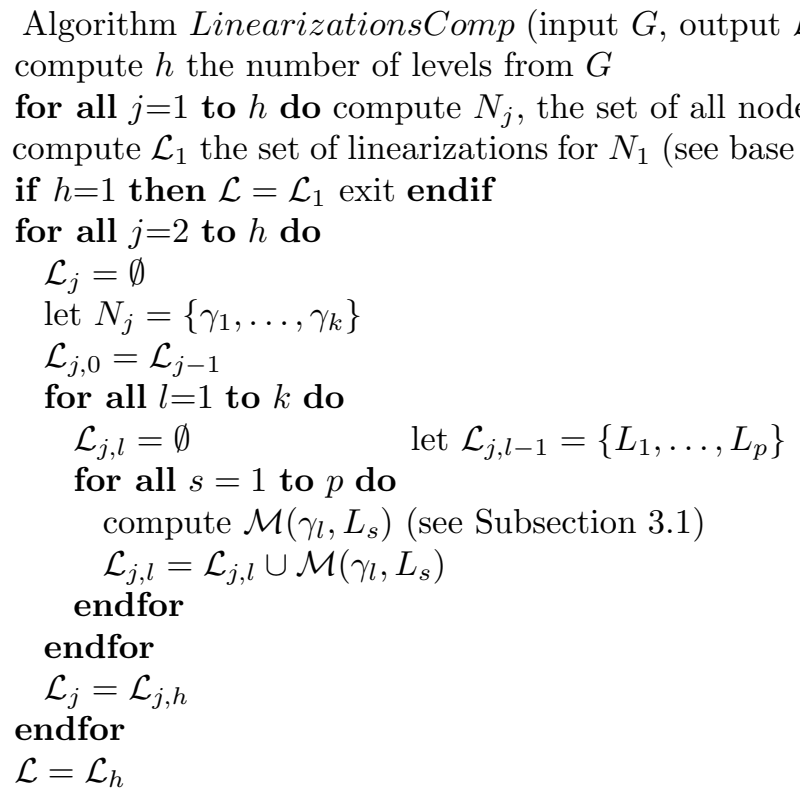

Secondly, we specify the computing of the linearizations corresponding to the expression $C$ that has comparison atoms as base expressions, and is formed of base atoms using the conjunction or disjunction operators. The expression $C$ is equivalent to a disjunction of conjunctions, i.e. $E \equiv E_{1} \vee \ldots \vee E_{p}$. For each expression $E_{j}$, we construct the forest $G_{E_{j}}$, and call the algorithm Linearizations Comp with the parameters $G_{E_{j}}$ and $\mathcal{L}_{j}$. The set of the linearizations for $C$, denoted $\mathcal{M}(C)$, is the union of all $\mathcal{L}_{j}$, i.e. $\mathcal{M}(C)=\cup_{j=1}^{p} \mathcal{L}_{j}$.

Example 4. Let us consider Example 2. Let $C$ be the comparison expression from $Q_{1}$. Since $z_{6} \geq c 2$ is equivalent to $\left(z_{6}>c 2\right) \vee\left(z_{6}=c_{2}\right)$, and $z_{6} \leq c_{3}$ is equivalent to $\left(z_{6}<c_{3}\right) \vee\left(z_{6}=c_{3}\right)$, the expression $C$ is equivalent to $C^{\prime \prime} \equiv \bigvee_{i=1}^{3}\left[\left(t_{1}=1\right) \wedge E_{i}\right]$ $\bigvee_{i=1}^{3}\left[\left(t_{1}=2\right) \wedge E_{i}\right]$, where $E_{1} \equiv\left(c_{2}<z_{6}\right) \wedge\left(z_{6}<c_{3}\right), E_{2} \equiv\left(c_{2}<z_{6}\right) \wedge\left(z_{6}=c_{3}\right)$, $E_{3} \equiv\left(z_{6}=c_{2}\right) \wedge\left(z_{6}<c_{3}\right)$. The expression $\left(z_{6}=c_{2}\right) \wedge\left(z_{6}=c_{3}\right)$ is non satisfiable because all constants are considered different. Concerning the constants $c_{2}$ and $c_{3}$, we assume $c_{2}<c_{3}$, otherwise the expression $C$ is not satisfiable. As we specified in Section 3, we represent a linearization as a set of groups, where a group is a linearization of all terms corresponding to the same value domain. For the group $\left\{z_{6}, c_{2}, c_{3}\right\}$, and the first conjunction from $C^{\prime \prime}$, we obtain one linearization: $c_{2}<z_{6}<c_{3}$. Hence, to the first conjunction of $C^{\prime \prime}$, we have two groups denoted $g_{1}, g_{2}$, where $g_{1} \equiv\left(t_{1}=1\right), g_{2} \equiv c_{2}<z_{6}<c_{3}$. We consider an order of linearizations as follows: $L_{1}$ for the first conjunction of $C^{\prime \prime}, L_{2}$ for the fourth, $L_{3}$ for the second, $L_{4}$ for the fifth, $L_{5}$ for the third, and $L_{6}$ for the sixth. Thus, the linearizations $L_{i}$ have the following forms: 


$$
\begin{aligned}
& L_{1} \equiv\left(t_{1}=1, c_{2}<z_{6}<c_{3}\right), L_{2} \equiv\left(t_{1}=2, c_{2}<z_{6}<c_{3}\right), \\
& L_{3} \equiv\left(t_{1}=1, z_{6}=c_{3}\right), L_{4} \equiv\left(t_{1}=2, z_{6}=c_{3}\right), \\
& L_{5} \equiv\left(t_{1}=1, z_{6}=c_{2}\right) \text { and } L_{6} \equiv\left(t_{1}=2, z_{6}=c_{2}\right) .
\end{aligned}
$$

\subsection{Complexity Issues}

Let us compute the time complexity of algorithm 1 . Let $G$ be a forest, with $n$ nodes and $p$ edges and $h$ its number of levels (corresponding to the depth of the forest) with $h \leq p+1$. For every level $j, 1 \leq j \leq h$, let $N_{j}$ be the number of nodes on level $j$. We have $\sum_{j=1}^{h} N_{j}=n$. The set $L_{j}$ from the algorithm gives all the linearizations obtained using nodes from levels 1 to $j$. The number of terms for each linearization from $L_{j}$ is $l e n g t h_{j}=\sum_{k=1}^{j} N_{k}$. Let us denote by $\left|L_{j}\right|$ the cardinal of the $L_{j}$ set. We have $\left|L_{1}\right|=N_{1}$ ! because there is no edge between the nodes on the first level. For the other levels, we have: $\left|L_{j+1}\right| \leq$ $N_{j+1} *$ length $_{j} *\left|L_{j}\right|, 1 \leq j \leq h-1$.

We obtain $\left|L_{h}\right| \leq N_{h} * N_{h-1} * \ldots * N_{1} *\left(N_{h-1}+\ldots+N_{1}\right) * \ldots *\left(N_{2}+N_{1}\right) * N_{1}$ !.

The set of terms can be grouped in classes, a class contains all terms having the same value domain. If $m$ is the maximum number of elements in these classes, then $m$ is the number of the forest nodes in the given algorithm.

\section{Conclusion}

In the paper, we propose an algorithm to compute linearizations and evaluate its complexity. These are useful in the process of constructing and proving the query equivalence between query expressed on the database schema and its rewritings using views. Queries and views considered here contain aggregate functions and arithmetic comparisons. Future work will focus on the aspects concerning using linearizations in the problem of queries equivalence when queries and views contain negations.

\section{References}

1. Afrati, F., Li, C., Mitra, P.: Rewriting queries using views in the presence of arithmetic comparisons. Theoretical Computer Science 368, 88-123 (2006)

2. Cohen, S.: Containment of aggregate queries. ACM SIGMOD 34(1), 77-85 (2005)

3. Cohen, S., Nutt, W., Serebrenik, A.: Rewriting aggregate queries using views. In: PODS, pp. 155-166 (1999)

4. Cohen, S., Nutt, W., Sagiv, Y.: Deciding equivalences among conjunctive aggregate queries. Journal of the ACM 54(2), 1-50 (2007)

5. Grumbach, S., Rafanelli, M., Shurin, S.: On the equivalence and rewriting of aggregate queries. Acta Informatica 4(8) (2004)

6. Heinzelman, W.R., Chandrakasan, A., Balakrishnan, H.: Energy-efficient Communication Protocol for Wireless Microsensor Networks. In: 33rd Annual Hawaii International Conference on System Sciences (HICSS-33), pp. 3005-3014 (2000)

7. Madden, S., Szewczyk, R., Franklin, M., Culler, D.: Supporting aggregate queries over ad-hoc wireless sensor networks. In: Proc. of 4th IEEE Workshop on Mobile Computing Systems and Applications, pp. 49-58 (2002) 